\title{
Glaucoma Referral Refinement in Ireland: Managing the Sensitivity-Specificity Paradox in Optometric Practice
}

\author{
Catriona Barrett \\ Technological University Dublin, catriona.barrett@tudublin.ie \\ O'Brien Colm \\ Oepartment of Ophthalmology. Mater Misericordiae University Hospital, Dublin, \\ James Loughman \\ Technological University Dublin, james.loughman@tudublin.ie
}

Follow this and additional works at: https://arrow.tudublin.ie/otpomart

Part of the Optometry Commons

\section{Recommended Citation}

Barrett, C., O'Brien, C. \& Loughman, J. (2018) Glaucoma Referral Refinement in Ireland: Managing the Sensitivity-Specificity Paradox in Optometric Practice,Ophthalmic Physiol Opt. 2018. httpsJ/doi.org/ I0.1111/opo. 12446

This Article is brought to you for free and open access by ARROW@TU Dublin. It has been accepted for inclusion in Articles by an authorized administrator of ARROW@TU Dublin. For more information, please contact arrow.admin@tudublin.ie, aisling.coyne@tudublin.ie, gerard.connolly@tudublin.ie.

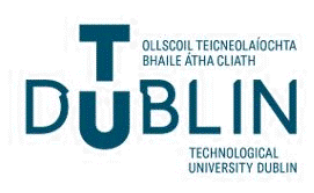




\begin{tabular}{|c|c|c|c|c|c|}
\hline 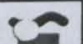 & O P O & 12446 & \multirow{2}{*}{ WILEY } & Dispatch: 12.2 .18 & CE: Jenifar Seles S \\
\hline 2 & Journal Code & Manuscript No. & & No. of pages: 11 & PE: Sakthivel R. \\
\hline
\end{tabular}

\title{
Glaucoma referral refinement in Ireland: managing the sensitivity-specificity paradox in optometric practice
}

\author{
21 Catriona Barrett ${ }^{1}+$ (D), Colm O'Brien ${ }^{2}$ and James Loughman ${ }^{1,3}$
}

'Department of Optometry, Dublin Institute of Technology, Dublin, 'Department of Ophthalmology, Mater Misericordiae University Hospital, Dublin,

3 Ireland, and ${ }^{3}$ African Vision Research Institute, University of KwaZulu-Natal, Durban, South Africa

Citation information: Barrett C, O'Brien C \& Loughman J. Glaucoma referral refinement in Ireland: managing the sensitivity-specificity paradox in optometric practice. Ophthalmic Physiol Opt 2018. https://doi.org/10.1111/opo.12446

Keywords: glaucoma, ophthalmology, optometry, patient management, referral, sensitivity and specificity

\section{Correspondence: Catriona Barrett} E-mail address: catriona.barrett@dit.ie

Received: 20 September 2017; Accepted: 24 January 2018

\begin{abstract}
Purpose: Glaucoma referral refinement (GRR) has proven a successful demand management strategy for glaucoma suspect cases in the United Kingdom (UK). A GRR clinic was established in Dublin, Ireland to investigate the clinical viability of this pathway outside the UK's National Health Service (NHS) structures, and away from the influence of National Institute for Clinical Excellence (NICE) guidance.

Methods: Glaucoma suspect patients were recruited into the scheme following referral from community optometrists in the greater Dublin area. The GRR exam protocol was designed in consultation with the participating ophthalmology department. The refinement scheme optometrist, trained through apprenticeship style experience at a hospital outpatient clinic, made a tentative management decision after carrying out the GRR exam. The final management decision was made in a 'virtual clinic' by a glaucoma specialist consultant ophthalmologist.

Results: Two hundred and twenty-five glaucoma suspect patients were seen in the scheme. After their first GRR visit, $28 \%$ were discharged back to their own optometrist, $42 \%$ were monitored in the GRR clinic, and $30 \%$ were referred to ophthalmology. After this monitoring cohort were further assessed, a total of $38 \%$ of the patients seen within the scheme required referral to ophthalmology. Sixteen percent of the total participant group $(n=225)$ were lost to follow up. Cohen's $\kappa$ was used to determine the level of agreement between the scheme optometrist and ophthalmologist. There was substantial agreement, with $\kappa=0.63$ for the first visit management decisions $(n=225)$. Agreement increased for subsequent monitoring visits with $\kappa=0.85$ for second visits $(n=65)$, and $\kappa=0.69$ for all management decisions within the scheme $(n=301)$. We received management outcomes for 44 of the 86 patients referred to ophthalmology. Of these $44,57 \%$ received medical treatment for glaucoma, $34 \%$ were monitored without treatment, $2 \%$ were discharged, and $7 \%$ had comorbidities that were assessed and managed.

Conclusion: Of the patients seen within the scheme, $62 \%$ did not require referral onward to ophthalmology, thus releasing the significant majority of hospital clinic slots that would previously have been required to examine such patients. The high level of inter-professional decision agreement likely reflects the benefits of prescheme apprenticeship style training and ongoing hospital clinic participation by the scheme optometrist. The rate of loss to follow up compares favourably with ophthalmology led, hospital based, glaucoma clinics. Nevertheless, the losses indicate that patient education remains a key priority for future planning.
\end{abstract}




\section{Introduction}

Optometrists play a vital role in the detection of glaucoma, the world's leading cause of irreversible blindness. ${ }^{1}$ Primary open angle glaucoma (POAG), the most common glaucoma sub-type ${ }^{2}$ is insidious, progressive and irreversible, presenting a significant public health challenge. ${ }^{2}$ As population screening for POAG detection is neither cost effective ${ }^{3}$ nor viable,${ }^{4}$ it is primarily detected through opportunistic case-finding during routine eye examinations. Evidence from the UK ${ }^{5}$ has shown that the vast majority of glaucoma and ocular hypertension cases are detected through opportunistic case-finding by community-based optometrists.

Glaucoma prevalence increases exponentially with increasing age. ${ }^{6}$ Continued population growth coupled with our increasing age profile $\mathrm{e}^{7}$ are accompanied, therefore, by a synchronous rise in the burden of care required for glaucoma and other age-related eye disease. In 2014, the number of people (aged 40-80 years) with glaucoma worldwide was estimated at 64.3 million, this is expected to increase to 76.0 million by 2020 and almost double to 111.8 million by $2040 .^{8}$

Advances in diagnostic and screening tools, such as automated perimetry, and changes in professional guidance ${ }^{9}$ with regard to glaucoma diagnosis and management protocols, also have the potential to increase the demand for glaucoma related care. Clinical guidelines are developed with the aim of improving the quality of care received by patients and ultimately, improving health outcomes. The ability of clinical guidelines to deliver on these aims is questionable, and while appropriate guidelines can be a useful tool for making care more consistent and efficient, flawed guidelines have the potential to cause harm to both patients and the healthcare system.

As an example, in April 2009, the National Institute for Health and Care Excellence (NICE) guidelines for glaucoma, 'Glaucoma: Diagnosis and management of chronic open angle glaucoma and ocular hypertension' were published in the UK. ${ }^{10}$ Although the scope of these guidelines did not include case-finding or screening for glaucoma, there was a requirement for all patients with 'repeatable pressures over $21 \mathrm{mmHg}$ by applanation tonometry to be assessed by a suitably trained healthcare professional with a specialist qualification and relevant experience'. This part of the guidance was interpreted as relevant to case-finding, and guidance was issued by a group of influential optometric organisations in the $\mathrm{UK}$, advising optometrists to refer patients with a repeated IOP reading of above $21 \mathrm{mmHg}$, regardless of any other clinical findings. ${ }^{11}$ This led to a rise in glaucoma suspect referrals to ophthalmology and a corresponding fall in the positive predictive value (PPV) of the referrals. ${ }^{12}$
Glaucoma referral refinement (GRR) schemes, which had emerged in the early 2000 s as a potential solution to the already high rate of false positive glaucoma referrals, ${ }^{13}$ proliferated in the UK after $2009,{ }^{14-17}$ largely in response to the rise in glaucoma referrals following the publication of the NICE guidance. GRR describes a two-tier assessment in which an initial suspicious finding is validated by a subsequent enhanced assessment. The aim is to increase the PPV of optometric referrals to ophthalmology services, which has been shown to be both clinically ${ }^{18}$ and financially ${ }^{16,19}$ viable within the National Health Service (NHS) system.

In Ireland, as with many countries, there are no specific clinical guidelines relating to glaucoma diagnosis or case finding in primary care. Although optometric referral patterns in Ireland have not been directly affected by NICE guidance, anecdotal evidence from the ophthalmology team within the Mater Misericordiae University Hospital (MMUH) in Dublin, indicates that the proportion of false positive glaucoma referrals is high. A recent multicentre review, ${ }^{20}$ analysing data from five tertiary referral centres across Europe, found that only $10 \%$ of all newly referred glaucoma suspect patients actually had glaucoma, confirming that this issue is common in many jurisdictions.

A number of factors contribute to the false positive glaucoma referrals from primary care services, including limited availability of diagnostic equipment and the relatively low prevalence of glaucoma among the population of patients seen in optometric practice. Overall POAG prevalence is estimated at $1.88 \%$, with prevalence rising to $3.2 \%$ in those aged over 70 years. $^{21}$ At this prevalence level, even tests with relatively high sensitivity and specificity will yield low PPVs. ${ }^{22,23}$ GRR provides a method of offering enhanced diagnostic testing to a cohort of glaucoma suspect patients. In this likely higher prevalence population, the available diagnostic tests will have better PPV. ${ }^{22,23}$

The need for demand management within Irish ophthalmology services is clear; figures for March 2017, show that 34675 individuals in the Republic of Ireland (total population 2016: 4.76 million $^{24}$ ) were on a waiting list for a first appointment at a consultant-led ophthalmology outpatient clinic, with 9309 individuals having spent 12 months or more on the waiting list. ${ }^{25}$ This echoes a pattern of systems overload that has been demonstrated in many developed. countries: the need for new, more collaborative care paradigms in the face of increased longevity and subsequent increased demand for eye care services has also been recognised in Australia ${ }^{26}$ and the US. ${ }^{27}$ Worldwide shortages of ophthalmologists ${ }^{28}$ are exacerbating this mismatch between capacity and demand. ${ }^{29}$ Strategic planning is needed if we are to deliver an improved service and avoid an increase in preventable visual impairment. This istudy was designed, therefore, to investigate the clinical viability of GRR outside 
the UK's NHS structures, and away from the influence of NICE guidance.

\section{Methods}

The project began as a collaboration between researchers and clinicians at the Dublin Institute of Technology (DIT) and the Mater Misericordiae University Hospital (MMUH) Dublin. It was agreed that a GRR scheme could be of benefit to the ophthalmology department and the National Optometry Centre (NOC) at DIT agreed to host the scheme. The project was established as part of a $\mathrm{PhD}$ project examining glaucoma case finding by optometrists in Ireland. The research student, being an optometrist, became the scheme's specialist optometrist in glaucoma (SOG). A 2 month period of pre-scheme training commenced in October 2011. This consisted of at home self-study and apprenticeship-style training through participation in consultant led hospital glaucoma clinics. This apprenticeship style training began with observation of senior medical staff and progressed towards independent examination of patients and case presentation by the SOG to the supervising consultant. This process of supervised practice was key to the development safe decision-making skills. There was no formal evaluation or assessment of the performance of the SOG before they began examining patients under the glaucoma referral refinement and monitoring service (GRRMS). The scheme began when the supervising ophthalmologist considered the SOG was ready, which was agreed after $24 \mathrm{~h}$ of training across six clinic sessions. It is recognised that this training and accreditation process would need to become more standardised if the scheme was to be expanded, but this arrangement was deemed appropriate for this process given that the detailed one to one supervision allowed the consultant to closely monitor and assess the SOG's ongoing performance. The SOG continued to attend one clinic session per week throughout the duration of the scheme, examining both glaucoma patients and suspects under the supervision of a glaucoma specialist consultant ophthalmic surgeon.

The pilot scheme was announced to Irish optometrists through email leaflets, a publication in the periodical journal of the Association of Optometrists Ireland (AOI), and a presentation at the AOI annual general meeting in November 2011. Glaucoma suspect patients were recruited into the scheme following referral from community based optometrists in the greater Dublin area. Optometrists were instructed that any new glaucoma referrals were eligible for the scheme though urgent cases should be directly referred to ophthalmology as usual. This study sought to investigate the clinical viability of GRR away from the influence of NICE guidance and the subsequent guidance from professional representative bodies in the $\mathrm{UK}^{30}$ therefore there was no specific guidance issued to community optometrists as to which clinical findings warranted referral to the GRRMS.

The purpose of the study was explained to each patient both verbally and through a written consent form. Only those who gave written consent to have their clinical information used in the study were included in the analysis.

The GRRMS exam was designed to include gold standard examination strategies; both $\mathrm{NICE}^{10}$ and European Glaucoma Society (EGS) guidelines ${ }^{31}$ were referred to in the process of drafting the examination protocol. This also resulted in the examination protocol aligning well with the current practice within the participating ophthalmology department, which provided reliable baseline information for patients that were referred to ophthalmology after the refinement exam. The agreed GRRMS exam included the following assessments:

1. Case history.

2. Anterior chamber slit lamp examination, including Redmond Smith and van Herick's techniques.

3. Goldmann tonometry.

4. Ultrasound pachymetry.

5. Visual field test (Humphrey Visual Field Analyser SITAFast 24-2).

6. Dilated, slit lamp indirect ophthalmoscopy exam.

7. Fundus photography.

While the refinement scheme optometrist made a preliminary management decision after the GRR exam, the final management decision was approved by a glaucoma specialist consultant ophthalmic surgeon, who acted as the scheme's reference standard. Digital fundus photographs, copies of the visual field plots, and a summary of the patient record which included case history information, slit lamp findings, intraocular pressure (IOP) and pachymetry readings, and the optometrist's written record of the optic disc assessment, were made available for the consultant to view in a 'virtual clinic', similar to that described by Trikha, et $a l^{14}$ and Kotecha, et al. ${ }^{32}$ following the patient's GRR exam. Patients were informed of their final management through phone calls from the scheme optometrist.

The clinical outcomes for the patients seen in the scheme were categorised into three broad groups:

1. Discharge from the GRR clinic back to the primary optometrist;

2. Monitor in the GRR clinic;

3. Refer to ophthalmology.

It was decided that clinical guidelines indicating specific clinical findings at which to refer, monitor or discharge would be either unmanageably large or harmfully oversimplified, and could not represent best practice for many individual patients. For clinical tests such as IOP: on cup-disc ratio (CDR) for example, there are no set values that can 
perfectly discriminate between early glaucoma and those who are non-glaucomatous. Thus, the scheme proceeded with no set protocols beyond defining the tests that should be carried out, and the clinicians made their management decisions after taking all of the relevant clinical findings into consideration.

The data collected were analysed on the software package for social sciences, IBM SPSS Statistics (www.ibm.com). A frequency analysis was run to determine the management outcomes within the scheme. One-way Welch's analysis of variance (ANOVA), Kruskal-Wallis $H$ Test and Cohen's kappa ( $\kappa)$ were used to further analyse the results.

\section{Results}

Two hundred and twenty-five patients were recruited into the scheme. The management outcomes are outlined in Table 1.

A proportion of those patients assigned to be monitored within the GRR clinic were lost to follow up. These were lost exclusively from the monitoring cohort who were not diagnosed with glaucoma but advised to continue regular monitoring of suspect features. Approximately one-third of those recalled dropped out at each monitoring interval. The exact figures are shown in Table 1 above. Overall, $16 \%$ of participants were lost to follow up.

Of the 225 patients seen within the scheme, 80 were referred to ophthalmology as glaucoma suspects, two of these 80 had comorbidities that were detected during the GRR exam, both choroidal naevi. A further six patients were confirmed as non-glaucomatous during their GRRMS exam though comorbidities were detected which were deemed to require referral. Of these six, two were ocular naevi and the remainder ranged in severity from a routine referral for medical management of severe blepharitis to a neuro-ophthalmology referral for suspect neurological field loss.

Table 1. Management outcomes from the Dublin GRR clinic. Percentages have been rounded to the nearest whole number resulting in some percentage totals differing from $100 \%$

\begin{tabular}{lllll}
\hline & $\begin{array}{l}\text { Visit 1 } \\
(n=225)\end{array}$ & $\begin{array}{l}\text { Visit 2 } \\
(n=95)\end{array}$ & $\begin{array}{l}\text { Visit 3 } \\
(n=16)\end{array}$ & $\begin{array}{l}\text { End of } \\
\text { study } \\
(n=225)\end{array}$ \\
\hline Discharge $n(\%)$ & $62(28 \%)$ & $34(36 \%)$ & $5(31 \%)$ & $101(45 \%)$ \\
Monitor $n(\%)$ & $95(42 \%)$ & $16(17 \%)$ & $3(19 \%)$ & $3(1 \%)$ \\
Refer $n(\%)$ & $64(28 \%)$ & $13(14 \%)$ & $3(19 \%)$ & $80(35.5 \%)$ \\
Refer & $4(2 \%)$ & $2(2 \%)$ & $0(0 \%)$ & $6(2.6 \%)$ \\
comorbidity $n(\%)$ & & $300^{\dagger}(32 \%)$ & $5(31 \%)$ & $35(16 \%)$ \\
Lost to & & & & \\
follow up $n(\%)$ & & & & \\
\hline
\end{tabular}

One patient in this cohort emigrated during the study and continued their care abroad.
Therefore, 86 patients were referred onwards from the scheme, $38 \%$ of the total group.

\section{Clinical variations between management groups}

A one-way ANOVA was conducted to determine if central corneal thickness (CCT), IOP, and vertical cup-disc ratio (vCDR) were different for the three core management groups based on the first visit management decision (discharge $n=66$, monitor $n=95$, refer $n=64$ ). In cases where the referral indicated bilateral suspicious findings, the more suspect eye was chosen as the study eye, or, if the clinical findings were equivalent in each eye, the study eye was randomly selected through a coin toss.

One outlier was removed from the CCT data as the patient had a pathologically thin cornea following previous ocular injury. Two more outliers were found, as assessed by inspection of a boxplot for values greater than 1.5 boxlengths from the edge of the box. However, these data points were kept in the analysis as they represented the wide range of CCT values present in a normal population. The CCT values were normally distributed, as assessed by visual inspection of the $\mathrm{Q}-\mathrm{Q}$ plots. There was homogeneity of variances, as assessed by Levene's test for equality of variances $(p=0.97)$. The differences in mean CCT between the management groups were not statistically significant, $F(2$, 221) $=1.382, p=0.25$ (see Table 2).

There were no outliers in the IOP data and values in each cohort were normally distributed, as assessed by visual inspection of boxplot and Normal Q-Q plots respectively. The assumption of homogeneity of variance was violated, as assessed by Levene's test for equality of variances ( $p=0.001)$. The difference between mean Goldmann IOP in the three management groups was statistically significant using Welch's ANOVA, Welch's $F(2,37.22)=129.21$, $p<0.0001$ (see Table 2). IOP increased from the discharge ( $n=66, M=16.26 \mathrm{mmHg}$, S.D. $=3.13$ ); to monitor ( $n=95, M=18.32 \mathrm{mmHg}$, S.D. $=3.47)$; to refer $(n=63$, $M=22.83 \mathrm{mmHg}$, S.D. $=5.22$ ) management groups, in that order (see Figure $1 b$ and Table 2). Games-Howell post hoc analysis revealed that the mean increase from the discharge to monitor groups $(2.06 \mathrm{mmHg}, 95 \% \mathrm{CI}[0.82$, $3.30])$ was statistically significant $(p<0.0001)$, as was the increase from monitor to refer $(4.51 \mathrm{mmHg}, 95 \%$ CI [2.73, 6.29], $p<0.0001$ ).

Welch's ANOVA was then repeated to determine if mean IOP was statistically significantly different for the three core management-groups based on the second visit management decision (discharge $n=35,1 \quad M=16.4 \mathrm{mmHg}$, S.D. $=2.2)$; (monitor $n=16, M=19.1$, S.D. $=4.6$ ); (refer $n=13, M=20.15$, S.D. $=5.2)$. The difference between

\footnotetext{
${ }^{1}$ One outlier was removed from this group.
} 
Table 2. A one-way ANOVA comparing the clinical findings for central corneal thickness (CCT2 intraocular pressure (IOP) ${ }^{\star}$ and vertical cup-disc ratio (vCDR) according to the first visit management group within the refinement clinic

\begin{tabular}{|c|c|c|c|c|}
\hline & Discharge $(n=66)$ & Monitor $(n=95)^{+}$ & Refer $(n=64)$ & $p$ value (ANOVA) \\
\hline $\begin{array}{l}\text { CCT } \\
\text { Mean (S.D.) }\end{array}$ & $570 \mu \mathrm{m}( \pm 39.63)$ & $569 \mu \mathrm{m}( \pm 38.41)$ & $560 \mu \mathrm{m}( \pm 40.06)$ & 0.25 \\
\hline $\begin{array}{l}\text { IOP } \\
\text { Mean (S.D.) }\end{array}$ & $16.26 \mathrm{mmHg}( \pm 3.13)$ & $18.32 \mathrm{mmHg}( \pm 3.47)$ & $22.83 \mathrm{mmHg}( \pm 5.22)$ & $<0.0001$ \\
\hline $\begin{array}{l}\text { vCDR } \\
\text { Mean (S.D.) }\end{array}$ & $0.38( \pm 0.17)$ & $0.48( \pm 0.17)$ & $0.54( \pm 0.18)$ & $<0.0001$ \\
\hline
\end{tabular}

*Welch's ANOVA.

4 'One outlier was removed from the CCT data, $n$ for the CCT monitoring cohort was 94

(a)

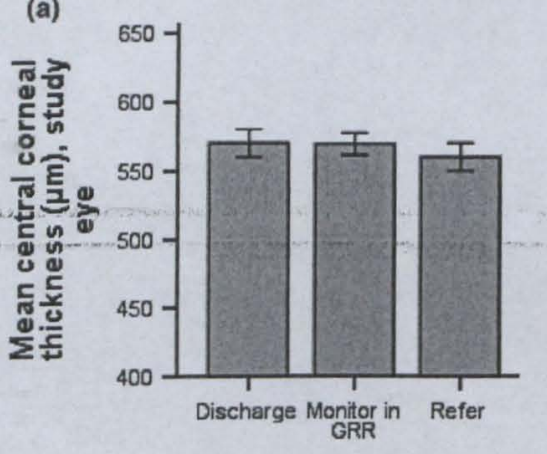

(b)

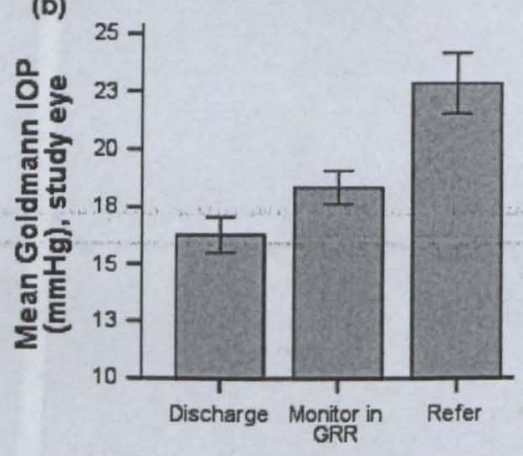

(c)

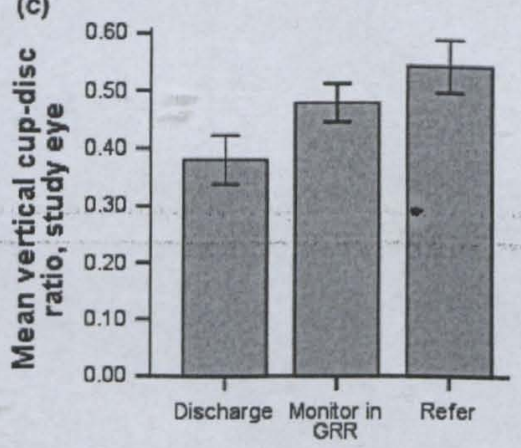

Management after first GRR visit

Error bars: $95 \% \mathrm{Cl}$

Figure 1. Mean values for central corneal thickness, intraocular pressure (IOP), and vertical cup-disc ratio (vCDR) in each first visit, refinement clinic management group (discharge $n=66$, monitor $n=95$, refer $n=64$ ).

mean second visit Goldmann IOP values in the three management groups was again found to be statistically significant, Welch's $F(2,20.50)=5.27, p=0.014$, but Games-Howell post hoc testing showed no statistically significant pairwise comparisons. These apparently conflicting results are due to the differences in the distributions used in the one-way ANOVA and the Games-Howell post hoc test and show that a statistically significant difference between groups is questionable.

There were no outliers in the VCDR data and values in each cohort were normally distributed, as assessed by visual inspection of boxplot and Normal Q-Q plots respectively. There was homogeneity of variances, as assessed by Levene' test for equality of variances $(p=0.45)$. There was a statistically significant difference between the three groups, $F(2$, 222 ) $=14.97, p<0.0001$ (see Table 2). vCDR increased from the discharge $(n=66, M=0.38$, S.D. $=0.17)$; to monitor $\left(n=95, M=0.48, S_{.}=0.17\right)$; to refer $(n=64$, $M=0.54$, S.D. $=0.18$ ) management groups, in that order (see Figure $1 c$ and Table 2). Tukey-Kramer post hoc analysis revealed that the increase from the discharge to monitor groups $(0.10,95 \% \mathrm{CI}[0.03,0.16])$ was statistically significant $(p=0.001)$, though the increase from monitor to refer
$(0.06,95 \%$ CI $[0.001,0.130], p=0.055)$ was not. The difference between the discharge and refer groups was significant at the $p<0.0001$ level $(0.16$, CI $[0.09,0.23])$.

The one way ANOVA was then repeated to determine if mean vCDR was statistically significantly different for the three core management groups based on the second visit management decision (discharge $n=36, M=0.50$, S.D. $=0.14$ ); (monitor $n=16, M=0.45$, S.D. $=0.19$ ); (refer $n=13, M=0.49, \mathrm{~S} . \mathrm{D} .=0.06$ ). The difference between mean second visit vCDR values in the three management groups was not found to be statistically significant, $F(2,23.70)=0.50, p=0.62$.

It was not possible to include visual field results in the ANOVA analysis as visual inspection of the normal Q-Q plots for all three global indices (visual field index (VFI), mean deviation (MD), and pattern standard deviation (PSD)) showed that the data was not normally distributed. For this non-parametric data, a Kruskal-Wallis $H$ Test was used to determine if there were significant differences between the medians of the three management groups. The PSD score was chosen as the global index most relevant to early glaucoma. Distributions of PSD scores were similar for all groups, as assessed by visual inspection of a boxplot. 
Median PSD scores were statistically significantly different between groups, $H(2)=11.251, p=0.004$. Pairwise comparisons were performed using Dunn's (1964) procedure with a Bonferroni correction for multiple comparisons. Statistical significance was accepted at the $p<0.0167$ level. This post hoc analysis revealed statistically significant differences in median PSD scores between the discharge (1.47) and refer (1.81) $(p=0.004)$ management groups, but just approached significance between the monitor (1.51) and refer groups $(p=0.024)$, and no significant difference was observed between the monitor and discharge groups $(p=1.00)$.

This test was then repeated to determine if the differences in median PSD score were still statistically significant for second visit management decisions (discharge $n=35$, monitor $n=16$, refer $n=19$ ) which showed that median PDS scores were not statistically significantly different between the groups, $H(2)=0.783, p=0.68$.

\section{Agreement between ophthalmologist and optometrist management decisions}

Cohen's kappa ( $\kappa$ ) was used to determine if there was agreement between the scheme optometrist and ophthalmologist. There was substantial agreement, ${ }^{33}$ with $\kappa \geq 0.63$ for all patient visits (Table 3).

The cross tabulation (Table 4) shows where the disagreements occurred.

Table 4 shows that there were 35 decisions where the scheme's reference standard, a glaucoma specialist consultant ophthalmic surgeon, had more conservative clinical management than theSOG.-These 35 decisions represent 33 patients as there were two occasions where disagreement was on the same patient at different visits. Of the 33 patients, seven were eventually discharged from the scheme, seven failed to return for their follow up appointments, and 19 were eventually referred to ophthalmology. Of these 19 , we were able to follow up on ophthalmology management outcomes for just seven patients: two were started on treatment, four were monitored in ophthalmology, and one was discharged. The two patients who received treatment in

Table 3. Inter-rater agreement within the virtual clinic

\begin{tabular}{|c|c|c|c|c|}
\hline & $\begin{array}{l}\text { Visit } 1 \\
(n=225)\end{array}$ & $\begin{array}{l}\text { Visit } 2 \\
(n=65)\end{array}$ & $\begin{array}{l}\text { Visit } 3 \\
(n=11)\end{array}$ & $\begin{array}{l}\text { All } \\
\text { management } \\
\text { decisions } \\
(n=301)\end{array}$ \\
\hline $\begin{array}{l}\text { Карра } \\
(95 \% \text { CI) }\end{array}$ & $\begin{array}{l}0.63 \\
(0.54-0.72)\end{array}$ & $\begin{array}{l}0.85 \\
(0.73-0.97)\end{array}$ & $\begin{array}{l}0.72 \\
(0.36-1.08)\end{array}$ & $\begin{array}{l}0.69 \\
(0.62-0.89)\end{array}$ \\
\hline$p$ & $p<0.0001$ & $p<0.0001$ & $p=0.001$ & $p<0.0001$ \\
\hline $\begin{array}{l}\text { Rate of } \\
\text { agreeme }\end{array}$ & $76.0 \%$ & $90.8 \%$ & $81.8 \%$ & $79.4 \%$ \\
\hline
\end{tabular}

ophthalmology had been marked for monitoring by the SOG. There was one occasion where a patient was marked for discharge by the SOG but subsequently referred to ophthalmology by the scheme's supervising ophthalmologist (Table 4), the management outcome for this patient was not available to us. We were able to follow up on management outcomes for 44 of the 86 patients referred to ophthalmology (Table 5).

\section{Discussion}

Only $38 \%$ of the patients seen in the scheme required referral for specialist hospital care demonstrating the scheme's significant potential to release capacity within hospital eye services. Those patients referred to ophthalmology had significantly improved clinical information, including full threshold visual fields on the Humphrey Visual Field Analyser, Goldmann tonometry readings, and ultrasound pachymetry measurements. Providing all of these tests within one GRR appointment creates a reliable baseline for future monitoring and negates the need for those patients to have separate appointments for different diagnostic tests such as visual field testing for example, which is often the case within the MMUH glaucoma clinic. With further training, the scheme could be further expanded to include optical coherence tomography (OCT) and gonioscopy so that the GRRMS could serve to provide best practice diagnostic testing for glaucoma suspects outside of the ophthalmology outpatient clinic, a model that has worked well elsewhere. $^{26}$

Of the eight co-morbidities detected in the scheme, four were retinal naevi. Future schemes should define a management protocol for this relatively common condition.

The first visit discharge rate $(29 \%)$ is similar to rates documented in the UK after the NICE guidelines, 'Glaucoma: Diagnosis and management of chronic open angle glaucoma and ocular hypertension' were published, ${ }^{16}$ an important finding in a jurisdiction that has no specific clinical guidelines relating to glaucoma diagnosis or case-finding. Sparrow ${ }^{34}$ argued that "hasty and ill-considered advice ... (to optometrists by influential professional bodies) ... produced an ongoing problem of unnecessary flooding of NHS glaucoma services, with false positive referrals frequently based on poor quality IOP measurements'. While there is truth in this statement, it is not the whole truth, as it places a distorted and arbitrary focus on false positive referrals and ignores the difficult role optometrists have in balancing their clinical judgement and their legal-responsibilities. Optometrists have a responsibility to detect disease during routine eye examination, which inherently leads to false positive referrals in a population where the relative prevalence of glaucoma is low. ${ }^{22}$ This effect is likely being compounded by a tendency for-optometrists to preference 
Table 4. Cross tabulation showing the optometrist's preliminary management decision (rows), and the final management decided by glaucoma consultant (columns)

\begin{tabular}{|c|c|c|c|c|c|}
\hline & & \multicolumn{4}{|c|}{ Final management decided by glaucoma consultant } \\
\hline & & Discharge & Refer & Monitor in GRR & Total \\
\hline \multirow[t]{3}{*}{ Optom decision } & Discharge & $83(78 \%)$ & 1 & $\underline{19}$ & 103 \\
\hline & Refer & 1 & $64(80 \%)$ & 3 & 68 \\
\hline & Monitor in GRR & 23 & $\underline{15}$ & $92(81 \%)$ & 130 \\
\hline Total & & 107 & 80 & 114 & 301 \\
\hline
\end{tabular}

Agreement is shaded in grey. Underlined figures represent occasions where the ophthalmologist was more conservative than the scheme optometrist.

Table 5. Management outcomes for patients referred to ophthalmology

\begin{tabular}{lrr}
\hline Management outcome & $\boldsymbol{n}$ & $\%$ \\
\hline Medical treatment & 25 & 57 \\
Monitored without treatment & 15 & 34 \\
Discharged at first visit & 1 & 2 \\
Managed co-morbidity & 3 & 7 \\
Total & 44 & 100 \\
\hline
\end{tabular}

sensitivity over specificity in their diagnostic testing, ${ }^{35}$ a practice pattern that could be considered pragmatic, given that optometrists are required to detect pathology and are at risk of litigation ${ }^{36,37}$ if they fail in this duty of care. Optometrists are faced with a paradoxical situation whereby rigorous, highly sensitive screening can often lower overall referral accuracy as it produces a high number of false positives, but the alternative, highly specific screening potentially increases the risk of missing disease that could lead to irreversible sight loss.

A number of approaches have failed to solve the problem of false positive glaucoma referrals. Vernon and $\mathrm{Ghosh}^{38}$ established that the provision of specific referral guidelines, circulated to all optometrists working within the catchment area, had little effect on the proportion of false positive referrals. Yoshioka, et al. ${ }^{39}$ showed that short-term didactic teaching programs had most effect on false negative rates in glaucoma referrals. This indicates that training may have a beneficial impact on the prevalence of undetected glaucoma, but is unlikely to significantly reduce false positives. GRR provided a safe method of offering enhanced diagnostic testing to a cohort of glaucoma suspects. In this likely higher prevalence population, the available diagnostic tests can produce better PPVs. ${ }^{23,40}$

Of course no medical test hàs perfect sensitivity and perfect specificity, and glaucoma detection is a particularly ambiguous area given the significant overlaps in the clinical features of suspicious, but normal individuals and those with early glaucoma. ${ }^{22,39}$ Accurate diagnosis of early glaucoma often requires careful monitoring until progression, the hallmark of glaucoma, can be identified or ruled out. ${ }^{41}$ Our results have highlighted the existence of a monitoring need in suspect glaucoma (Table 1), and careful consideration should be given to how this cohort of patients can be best served.

Recent changes in the legislation governing optometric practice in Ireland, has framed scope of practice more broadly. ${ }^{42}$ This has created the potential for optometrists to become more involved in the diagnosis, monitoring and management of ocular pathology, as has happened in many other jurisdictions including Australia, ${ }^{43}$ the $\mathrm{UK}^{44,45}$ and the USA. ${ }^{46}$

There is a skills and experience gap however, in moving from a screening role to an enhanced diagnostic or management role: a recent survey about the barriers perceived by optometrists in relation to glaucoma care found a majority of Irish optometrists agreed that a lack of training limited their ability to detect glaucoma during routine eye exams. ${ }^{47}$ Our collaborative care scheme allowed for optometric skill and equipment to be utilised in collaboration with ophthalmology expertise and experience, delivering better access to expert care, while ongoing hospital-based apprenticeship style training for the scheme optometrist facilitated real improvements in optometric clinical skill, which cannot be achieved through didactic training programmes alone. ${ }^{39}$

The level of inter-observer agreement $(\kappa \geq 0.63$ for all patient visits, Table 3) was substantial, which likely reflects the benefits of pre-scheme apprenticeship style training and ongoing hospital clinic participation by the scheme optometrist, which ensured adequate glaucoma experience, while also facilitating communication between optometry and ophthalmology, as recommended by Lockwood, et $a .^{18}$ and Trikha, et al. ${ }^{14} \mathrm{~A}$ higher level of agreement was achieved for those who were monitored ( $\kappa=0.85$ for second visit management decisions, Table 3 ). This aligns with the findings from Wright and Diamond ${ }^{48}$ who observed a kappa value of 0.69 for monitoring reviews of glaucoma patients and suspects. 
Some amount of disagreement in relation to glaucoma is to be expected from the scheme. It is well recognised that decision making algorithms in glaucoma are complex, and that even glaucoma specialist ophthalmic consultants exhibit a wide range of agreement with each other, and even themselves, when diagnosing or managing the condition. ${ }^{49}$ The most common area of disagreement was between the discharge and monitor groups, likely due to the ambiguity in these suspect cases. There was one occasion where a patient was non-conservatively marked for discharge by the optometrist but subsequently referred to ophthalmology by the scheme's supervising ophthalmologist. Although the management outcome was not available to us for this isolated case, this example does highlight the advantage of close inter-professional cooperation and the utility of virtual clinic reviews in ensuring patient safety in the scheme.

Substantial agreement was achievable despite the lack of predefined management protocols within the scheme. The clinical measurements for IOP, vCDR and PSD showed statistically significant differences between first visit management groups but the observed differences just failed to reach statistical significance at the second visit. It is possible that a larger sample size in the second visit cohort would have achieved statistical significance as the data is trending in this direction. This perhaps confirms that guidelines may be broadly applicable to a large cohort of patients, but not appropriate in many individual cases, particularly more ambiguous presentations that require monitoring. Alternatively, these results could represent the historical reliance on these measurements in glaucoma diagnosis. It is currently understood that these markers are not sufficient to characterise such a complex disease. Thus, clinical judgement needs to supersede guidelines at times. In fact, the diagnostic criteria for glaucoma have varied widely between studies: Wolfs, et al..$^{50}$ estimated that the overall prevalence of POAG may vary up to 12 -fold with different criteria and screening algorithms.

It is notable that $33 \%$ of those we were able to follow up in ophthalmology were monitored without treatment (Table 5) even when a glaucoma subspecialist recommended they were referred (essentially a false positive ophthalmology referral, which provides further evidence as to the difficulty in finding the right sensitivity-specificity balance). This reflects the gap between the sensitivity required when screening for glaucoma and the specificity required when making decisions regarding treatment. We believe that the GRRMS provides a way to manage this sensitivityspecificity paradox, which may not be achievable by other means. In fact, emphasis on false positive referrals could create a culture of diminishing sensitivity, where optometric glaucoma referrals are very specific, but glaucoma diagnoses are missed because of reluctance to refer or inability to carry out appropriate follow up investigations.
Approximately $50 \%$ of those with glaucoma in Ireland ${ }^{21}$ and other developed countries ${ }^{51}$ are unaware of their disease. To reduce visual impairment and thus loss of independence in the aging population, detection of OAG is of utmost importance. Rather than placing arbitrary focus on false positive referrals, the scheme facilitated open communication between those screening for the disease and those responsible for treatment, as well as clearer acknowledgement and planning for the necessary work of monitoring suspect cases.

On first review, the dropout rate (approximately onethird of patients in the monitoring group, see Table 1) may be a cause for concern. However, the rate of loss to follow up actually compares favourably with ophthalmology led, hospital based glaucoma clinics, ${ }^{52}$ demonstrating a potential advantage of community based care. A similar loss to follow up for glaucoma suspect patients was reported in an optometry-led collaborative glaucoma care scheme in Australia. ${ }^{26}$ It has been documented that glaucoma suspects are significantly more likely to drop out of follow up compared to those with established glaucoma ${ }^{53}$ and that patients' understanding of glaucoma disease mechanisms, including the insidious and irreversible nature of the condition, has been shown to greatly influence their adherence to recommended follow-up visits. ${ }^{54}$ This indicates that improved patient education and emphasis on good physician-patient communication should be a key priority for future planning.

\section{Limitations}

The voluntary nature of the study could have affected the sample of referrals obtained. The scheme was established through voluntary participation from optometrists and patients within the greater Dublin area. As referral to this scheme was optional, optometrists may have referred more highly suspect patients to ophthalmology preferentially, seeing referral to a refinement clinic as unnecessary when they were certain of their diagnosis. Access to all glaucoma referrals during the study time period would likely better represent the true nature of optometric referrals in Ireland. It should be noted however, that the referrals did represent a broad spectrum of glaucoma, from early to advanced stages.

Lack of access to ophthalmology patient records also limited the documentation of the final ophthalmology management outcomes for the referred patients and made it impossible to assess the positive predictive rate (PPR) of the refined referrals. The scheme operated with cooperation from the glaucoma team at the MMUH, so we were ableito access outcomes for the cohort of patients that were subsequently seen in the outpatient glaucoma clinic at the MMUH. Some of the patients referred into the scheme 
were not within the MMUH catchment area. If these patients required referral to ophthalmology, they were sent to the appropriate public ophthalmology service, or if they so wished, to a suitable private ophthalmology service. Feedback from these services was difficult to attain, it was dependent on individual doctors within the services sending a response back to the SOG. Historically, optometry has remained a community-based profession in Ireland, and not been integrated into hospital services. Therefore, optometrists typically have no access to hospital eye service records and only sporadic feedback from the public ophthalmology services to which they refer. Initial findings indicate a high level of accuracy within the refined referrals, with only one patient being discharged from ophthalmological review at first visit. The PPV of GRR schemes has previously been calculated at $0.78,{ }^{14}$ a marked improvement over unrefined glaucoma referrals $(0.37) .{ }^{18}$ Further work needs to be done on the follow up of glaucoma referrals within an Irish hospital eye service. Determining the PPV for both refined and unrefined optometric glaucoma referrals in Ireland would give more insight into the value of the Dublin GRRMS.

The agreement rate between clinicians was high, but the scheme involved just one ophthalmologist and one optometrist. It might not be possible to achieve this level of agreement once the scheme is expanded. Furthermore, the scheme optometrist was involved in this project as part of their $\mathrm{PhD}$ research and therefore may have been more invested in the training and literature review than might be typical of most community based optometrists. Future work should continue to assess inter-practitioner agreement to determine whether agreement remains high when multiple clinicians are employed.

The false negative rate of the scheme was not assessed. It is possible that some true glaucoma cases were discharged from the scheme. All patients who were discharged from the GRRMS were advised to visit their optometrist for annual or biennial eye exams in the future and a detailed report of the GRRMS findings was sent to the referring optometrist. The false negative rate from similar schemes has been reassuringly low, ${ }^{40,55}$ though the sample sizes in these false negative studies have also been low, leaving some uncertainty regarding the true false negative rate of GRR schemes. Any expansion of the Dublin GRRMS should look to incorporate a mechanism to assess false negatives.

While similar initiatives have produced substantial cost savings for the state, ${ }^{14,17}$ future work should provide an economic evaluation of the seheme. The cost effectiveness of GRR schemes has been shown to vary significantly ${ }^{56}$ depending on the financial models used. The national average cost of an outpatient visit in Ireland was estimated to be $€ 130$ in 2011 using a top-down methodology (National Casemix Programme) however, no information is available on how this cost may vary across specialties. This is approximately treble the amount currently paid by the State for dilated eye examinations conducted by community optometrists $(€ 45)$. Costing an outpatient ophthalmology clinic appointment will be an important step in assessing the financial viability of any community based ophthalmic shared-care scheme in Ireland, but it appears likely that such a scheme could generate cost savings.

\section{Conclusions}

The GRRMS proved a safe and effective collaboration between optometry and ophthalmology, facilitating community refinement and monitoring of the majority of glaucoma suspect patients. Current waiting times for state funded ophthalmology-led clinic appointments are at unacceptable levels, in excess of 18 months in some hospitals, ${ }^{25}$ leaving vulnerable patients at risk of permanent sight loss. The monitoring facility in this scheme acted to bridge the gap between the sensitivity required when case finding for glaucoma and the specificity required when initiating treatment. This pilot scheme confirms that there is potential for GRRMS to release capacity within hospital outpatient clinics, although we cannot be sure what affect this might have on waiting lists until a larger scheme is implemented. Pending economic evaluation, state agencies should consider how care structures could be modified to support further development of GRRMS in Ireland.

\section{Acknowledgements}

This work was supported by research funding from the Association of Optometrists Ireland.

\section{Disclosure}

The authors report no conflicts of interest and have no proprietary interest in any of the materials mentioned in this article.

\section{References}

1. Resnikoff S, Pascolini D, Etya'ale D et al. Policy and practice. Bull World Health Organ 2004; 82: 844-851.

2. Quigley $\mathrm{H} \&$ Broman $T$. The number of people with glaucoma worldwide in 2010 and 2020. Br J Ophthalmol 2006; 90: 262-267.

3. Burr JM, Mowatt G, Hernández Ret al. The clinical effectiveness and cost-effectiveness of screening for open angle glaucoma: a systematic review and economic evaluation. Health Technol Assess 2007; 11: 1-190.

4. Mowatt G, Burr JM, Cook JA et al. Screening tests for detecting open-angle glaucoma: Systematic review and 
meta-analysis. Invest Ophthalmol Vis Sci 2008; 49: 53735385.

5. Bowling B, Chen SDM \& Salmon JF. Outcomes of referrals by community optometrists to a hospital glaucoma service. BrJ Ophthalmol 2005; 89: 1102-1104.

6. Broman AT, Quigley HA, West SK et al. Estimating the rate of progressive visual field damage in those with open-angle glaucoma, from cross-sectional data. Invest Ophthalmol Vis Sci 2008; 49: 66-76.

7. Layte $\mathrm{R}, \mathrm{Barry} \mathrm{M}$, Bennett $\mathrm{K}$ et al. Projecting the impact of demographic change on the demand for and delivery of health care in Ireland. Economic and Social Research Institute, Dublin, 2009. https://www.esri.ie/publications/projectingthe-impact-of-demographic-change-on-the-demand-forand-delivery-of-health-care-in-ireland/, accessed 20/9/17.

8. Tham YC, Li X, Wong TY, Quigley HA, Aung T \& Cheng $\mathrm{CY}$. Global prevalence of glaucoma and projections of glaucoma burden through 2040: a systematic review and metaanalysis. Ophthalmology 2014; 121: 2081-2090.

9. Association of Optometrists Ireland. Guidelines for Members regarding performance of Eye Examinations. 2009. http:// www.aoi.ie/system/files/attachments/Eye\%20Examination\% 20Guidelines\%20Aug\%2009_0.pdf, accessed 20/9/17.

10. National Institute for Health and Care Excellence (NICE). Glaucoma: diagnosis and management | Guidance and guidelines | NICE. https://www.nice.org.uk/guidance/cg85, accessed 10/9/17.

11. Vernon SA, Hillman JG, Macnab HK et al. Community optometrist referral of those aged 65 and over for raised IOP post-NICE: AOP guidance versus joint college guidance-an epidemiological model using BEAP. Br J Ophthalmol 2011; 95: 1534-1536.

12. Shah $S$ \& Murdoch IE. NICE - impact on glaucoma case detection. Ophthalmic Physiol Opt 2011; 31: 339-342.

13. Henson DB, Spencer AF, Harper R \& Cadman EJ. Community refinement of glaucoma referrals. Eye (Lond) 2003; 17: 21-26.

14. Trikha S, Macgregor C, Jeffery M \& Kirwan J. The Portsmouth-based glaucoma refinement scheme: a role for virtual clinics in the future? Eye (Lond) 2012; 26: 1288-1294.

15. Bourne RR, French $K$, Chang $L$, Borman $A D$, Hingorani $M$ \& Newsom WD. Can a community optometrist-based referral refinement scheme reduce false-positive glaucoma hospital referrals without compromising quality of care? The community and hospital allied network glaucoma evaluation scheme (CHANGES). Eye (Lond) 2010; 24: 881-887.

16. Ratnarajan G, Newsom W, Vernon $S$ et al. The effectiveness of schemes that refine referrals between primary and secondary care-the UK experience with glaucoma referrals: the Health-Innovation \& Education Cluster (HIEC) Glaucoma Pathways Project. BMJ Open 2013; 3: 1-8.

17. Devarajan N, Williams GS, Hopes M, O'Sullivan D \& Jones D. The Carmarthenshire Glaucoma Referral Refinement Scheme, a safe and efficient screening service. Eye (Lond) 2011; 25: 43-49.
18. Lockwood AJ, Kirwan JF \& Ashleigh Z. Optometrists referrals for glaucoma assessment: a prospective survey of clinical data and outcomes. Eye (Lond) 2010; 24:1515-1519.

19. College of Optometrists and College of Ophthalmologists. Commissioning better eye care: Glaucoma. 2013. https://www. rcophth.ac.uk/wp-content/uploads/2014/12/Low-vision-

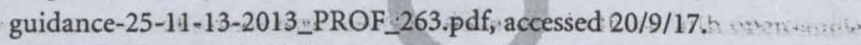

20. Founti $\mathrm{P}$, Topouzis F, Holló G et al. Prospective study of glaucoma referrals across Europe: are we using resources wisely? Published Online First: 03 August 2017. https://doi. org/10.1136/bjophthalmol-2017-310249, accessed 20/9/17.

21. Coffey M, Reidy A, Wormald R, Xian WX, Wright L \& Courtney P. Prevalence of glaucoma in the west of Ireland. BrJ Ophthalmol 1993; 77: 17-21.

22. Harper R, Henson D \& Reeves BC. Appraising evaluations of screening/diagnostic tests: the importance of the study populations. Br J Ophthalmol 2000; 84: 1198-1202.

23. Grimes DA \& Schulz KF. Uses and abuses of screening tests. Lancet 2002; 359: 881-884.

24. Central Statistics Office. Census 2016 Summary Results - Part 1. 2017. http://www.cso.ie/en/csolatestnews/presspages/ 2017/census2016summaryresults-part1/, accessed 20/9/17.

25. National Treatment Purchase Fund. Outpatient waiting list by specialty. http://www.ntpf.ie/home/outpatient.htm, accessed 19/4/17.

26. Jamous KF, Kalloniatis M, Hennessy MP, Agar A, Hayen A \& Zangerl B. Clinical model assisting with the collaborative care of glaucoma patients and suspects. Clin Exp Ophthalmol 2015; 43: 308-319.

27. Mets MB, Iii WLR, Lee P, Schuman JS \& Wilson D. The ophthalmic practice of the future. Arch Ophthalmol 2012; 130: 1195-1198.

28. Resnikoff S, Felch W, Gauthier T \& Spivey B. The number of ophthalmologists in practice and training worldwide : a growing gap despite more than 200000 practitioners. $\mathrm{Br} J$ Ophthalmol 2012; 96: 783-788.

29. Smith R. Our ophthalmology service is 'failing' - please help. The Royal College of Ophthalmologists Professional Standards Commitee 15/08/2013. https://www.rcophth.ac.uk/ 2013/08/our-ophthalmology-service-is-failing-please-help/, accessed 30/6/17.

30. Association of British Dispensing Opticians, Association of Optometrists, Federation of Ophthalmic and Dispensing Opticians. Advice on NICE glaucoma guidelines. London, 2009. www.assoc-optometrists.org/uploaded_ files/glaucoma_advice_and_faqs_01.06.09.pdf, accessed 22/ $3 / 17$.

31. European Glaucoma Society. Terminology and guidelines for glaucoma, 3rd edition. 2008. http://www.eyenet.gr/wp-conte nt/uploads/2010/01/EGS_Guidelines_English1.pdf, accessed 28/11/17.

32. Kotecha A, Longstaff S, Azuara-Blanco A et al. Developing standards for the development of glaucoma virtual clinics using a modified Delphi approach. Br J Ophthalmol 2017; ????: ????-????. bjophthalmol-2017-310504. 
33. Landis JR \& Koch GG. The measurement of observer agreement for categorical data. Biometrics 1977; 33: 159-174.

34. Sparrow JM. How nice is NICE? Br J Ophthalmol 2013; 97: 116-117.

35. Hadwin SE, Redmond T, Garway-Heath DF et al. Assessment of optic disc photographs for glaucoma by UK optometrists: The Moorfields Optic Disc-Assessment * Study (MODAS). Ophthalmic Physiol Opt 2013; 33: 618-624.

36. Meszaros L. Navigating optometric litigation. Optom. Times. 2012. http://optometrytimes.modernmedicine.com/optom etrytimes/news/modernmedicine/modern-medicine-featurearticles/navigating-optometric-litigation?page $=$ full, accessed 22/3/17.

37. Sherman J. Top Triggers for Malpractice Suits. Optom. Manag. 2001. http://www.optometricmanagement.com/is sues/2001/July-2001/top-triggers-for-malpractice-suits, accessed 22/3/17.

38. Vernon $S$ \& Adair A. Shared care in glaucoma: a national study of secondary care lead schemes in England. Eye (Lond) 2010; 24: 265-269.

39. Yoshioka N, Wong E, Kalloniatis M et al. Influence of education and diagnostic modes on glaucoma assessment by optometrists. Ophthalmic Physiol Opt 2015; 35: 682-698.

40. Kotecha A, Brookes J \& Foster PJ. A technician-delivered 'virtual clinic 'for triaging low-risk glaucoma referrals. Eye (Lond) 2017; 31: 899-905.

41. Heijl A, Leske CM, Bengtsson B, Hyman L, Bengtsson B \& Hussein M. Reduction of intraocular pressure and glaucoma progression. Arch Ophthalmol 2002; 120: 1268-1279.

42. Health (Miscellaneous Provisions) Act 2015. http://www.irish statutebook.ie/eli/2014/act/33/enacted/en/html, accessed $20 /$ 9/17.

43. Kiely PM, Cappuccio S \& McIntyre E. Optometry Australia scope of practice survey 2015. Clin Exp Optom 2017; 100: 260-269.

44. Harper R, Creer R, Jackson J et al. Scope of practice of optometrists working in the UK Hospital Eye Service: a national survey. Ophthalmic Physiol Opt 2016; 36: 197-206.

45. Needle JJ, Petchey R \& Lawrenson JG. A survey of the scope of therapeutic practice by UK optometrists and their attitudes to an extended prescribing role. Ophthalmic Physiol Opt 2008; 28: 193-203.

46. Nguyen Q \& Geller M. Optometry scope of practice in the United States. http://www.newgradoptometry.com/optom etry-scope-of-practice-united-states/, accessed 3/6/17.

47. Barrett C, Butler J, O'Brien C \& Loughman J. Barriers to glaucoma case findingastperceived by optometrists in Ireland. Clin Exp Optom 2017; ????: 1-10. https://doi.org/10. 1111/cxo.12573, accessed 20/9/17.

48. Wright HR \& Diamond JP. Service innovation in glaucoma management: using a web-based electronic patient record to facilitate virtual specialist supervision of a shared care glaucoma programme. Br J Ophthalmol 2015; 99: 313-317.

49. Murdoch I \& Johnston RL. Consultant clinical decision making in a glaucoma clinic. Eye (Lond) 2009; 24: 10281030.

50. Wolfs RCW, Borger PH, Ramrattan RS et al. Changing views on open-angle glaucoma : definitions and prevalences - The Rotterdam Study. Invest Ophthalmol Vis Sci 2000; 41:3309-3321:

51. Quigley HA. Number of people with glaucoma worldwide. Br J Ophthalmol 1996; 80: 389-393.

52. Ashaye AO \& Adeoye AO. Characteristics of patients who dropout from a glaucoma clinic. J Glaucoma 2008; 17: 227232.

53. Kosoko O, Quigley HA, Vitale S, Enger C, Kerrigan L \& Tielsch JM. Risk factors for noncompliance with glaucoma follow-up visits in a residents' eye clinic. Community Eye Health J 1998; 11: 62.

54. Murakami Y, Lee BW, Duncan M et al. Racial and ethnic disparities in adherence to glaucoma follow-up visits in a county hospital population. Arch Ophthalmol 2011; 129: 872-878.

55. Ratnarajan G, Kean J, French K, Parker M \& Bourne R. The false negative rate and the role for virtual review in a nationally evaluated glaucoma referral refinement scheme. Ophthalmic Physiol Opt 2015; 35: 577-581.

56. Baker H, Ratnarajan G, Harper RA, Edgar DF \& Lawrenson JG. Effectiveness of UK optometric enhanced eye care services: a realist review of the literature. Ophthalmic Physiol Opt 2016; 36: 545-557. 